\title{
How should we assess the mechanical properties of lower-limb prosthesis technology used in elite sport?-An initial investigation
}

\author{
Bryce Dyer, Philip Sewell, Siamak Noroozi \\ School of Design Engineering \& Computing, Bournemouth University, Poole, UK \\ Email: brdyer@bournemouth.ac.uk
}

Received 13 December 2012; revised 12 January 2013; accepted 18 January 2013

\begin{abstract}
Despite recent controversy, it is not yet formally recognised how lower-limb prosthesis should be assessed for their performance. To assist in this process, experiments are undertaken to investigate the linearity, stiffness and assessment of feet based energy return prosthesis technology typically used for elite level high speed running. Through initial investigations, it is concluded that static load testing would not be recommended to specify or regulate energy return prostheses for athletes with a lower-limb amputation. Furthermore, an assessment of energy return technology when loaded under dynamic conditions demonstrates changes in mechanical stiffness due to bending and effective blade length variation during motion. Such radical changes of boundary conditions due to loading suggest that any assessment of lower-limb prosthesis technology in the future should use methods that do not assume linear mechanical stiffness. The research into such effects warrants further investigation in the future.
\end{abstract}

Keywords: Prostheses; Stiffness; Running; Amputee; Non-Linearity

\section{INTRODUCTION}

Paralympic based competition has seen major changes in its use of technology from its inception uptil the modern day. The introduction of the Seattle foot in 1981 demonstrated the use of energy storing prosthetic feet in clinical prescription [1]. This comprised a flexible keel housed inside a polyurethane shell. When loaded, energy is retained within the structure as potential energy and a percentage of this is then returned to the user to assist their walking motion. However, extending from this development, a significant advance for both above and below knee amputees was made when Van Philips conceived the Flexfoot in 1987 [1]. This design is the basis of cur- rent sports lower-limb prosthesis technology which first saw use in elite competition at the 1988 Paralympic Games [2].

Lower-limb sports prostheses have been proposed as being passive forms of technology that should not surpass the performance of the limb they have replaced [3]. However, during the 2012 Paralympic Games $200 \mathrm{~m}$ event, Oscar Pistorius claimed that fellow competitor Alan Oliveira had a technological advantage through a change in the design of the lower-limb prosthesis that he had recently been using. As a result, a prediction of potential unfairness within disability sport amputee sprinting [4] now seems to being realised.

The key performance indicators of sprinting have been proposed as ground reaction force which then directly influences step frequency and stride length [5]. In terms of the characteristics of elite level runners, high lowerlimb stiffness is also critical and is predominantly provided by the knee joint [6]. With a non-disabled participant, it has been reported that stiffness of the lower-limb typically remains the same upto moderate running speeds due to the leg spring length changing to compensate [7] and has been show to increase with higher speeds [8]. However, in the case of lower-limb sprinters with a below-knee amputation, it is assumed that the disabled runner cannot modulate lower-limb stiffness to the same degree due to the passive nature of prosthesis and a loss of knee and/or the ankle.

Multiple methods have previously been used to assess the contribution of lower-limb prosthesis technology and these have been applied to an elite bi-lateral amputee athlete [9]. However, this study used predominately physiological outcome measures to determine the technological contribution of such technology. Dyer et al. concluded that when evaluating lower-limb sports prosthesis, a mechanical, and equipment focused approach to measuring performance enhancement was ethically more desirable [3].

A biological lower-limb is a highly dynamic, stiffness 
adjusting entity [7]. However, because an energy return prosthesis is inherently a spring, it could be assumed that when subjected to force, its performance can be calculated as a force-displacement curve [1] or that of a constant stiffness [2]. However, dynamic variability in the lower-limb stiffness of amputee prostheses when running have been recorded [10]. If controversy over such technology continues in sport and functional regulation of such technology is ultimately required [3], decisions based upon the assumption of lower-limb prosthesis linear response or assessment requires further investigation.

This paper attempts to initially investigate the following research questions:

1) Is static load assessment suitable to assist in the specification of energy return based prosthesis used for competitive running?

2) Is a prediction of energy return technology possible using static load techniques?

3) Are lower-limb foot prosthesis potentially subjected to significant boundary condition changes due to loading?

\section{METHODS}

Three pilot investigations are undertaken to initially investigate this papers research questions. This will be attempted using three different methods:

1) The linearity characteristic implications of energy return prosthesis are assessed when using two different point contact loading techniques of a statically applied load;

2) A $15 \mathrm{Kg}$ drop test of an energy return prosthesis from a $110 \mathrm{~mm}$ height to see if the prosthesis point of contact changes when subjected to a dynamic impact;

3) A qualitative assessment of steady state run tests using energy return technology.

An "Elite Blade" composite energy return foot prosthesis (Chas A Blatchford \& Sons Ltd, Basingstoke, UK) is used for the purpose of tests a and $b$. This prosthesis is shown in Figure 1. This prosthesis is designed to undertake a range of activity including low speed running of a user having a mass of circa $55 \mathrm{Kg}$. This prosthetic "blade" is not intended for running speeds expected for elite athletes. However, in principle, it is essentially uses identical energy absorption and return technology as more specific sprinting designs.

For test c, energy return footwear (Tramp-it BV, Den Haag, The Netherlands) was used to simulate energy storage and return technology. This is shown in Figure 2.

The footwear comprises a compound curved steel spring. Due to the footwear's extensive strapping, this does restrict the participant's ankle joint range of motion but it is conceded that it will not remove the joints contribution altogether. Use of this footwear is purely designed to demonstrate the characteristics of energy return

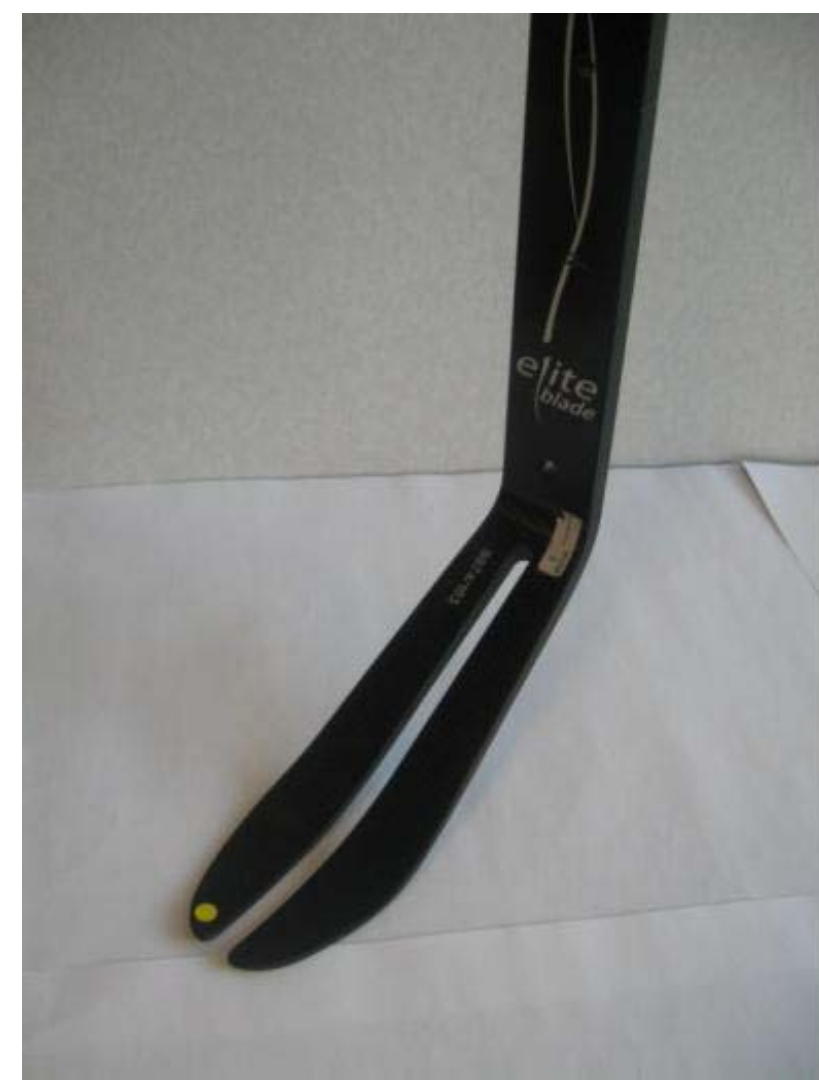

Figure 1. Elite blade prosthesis.

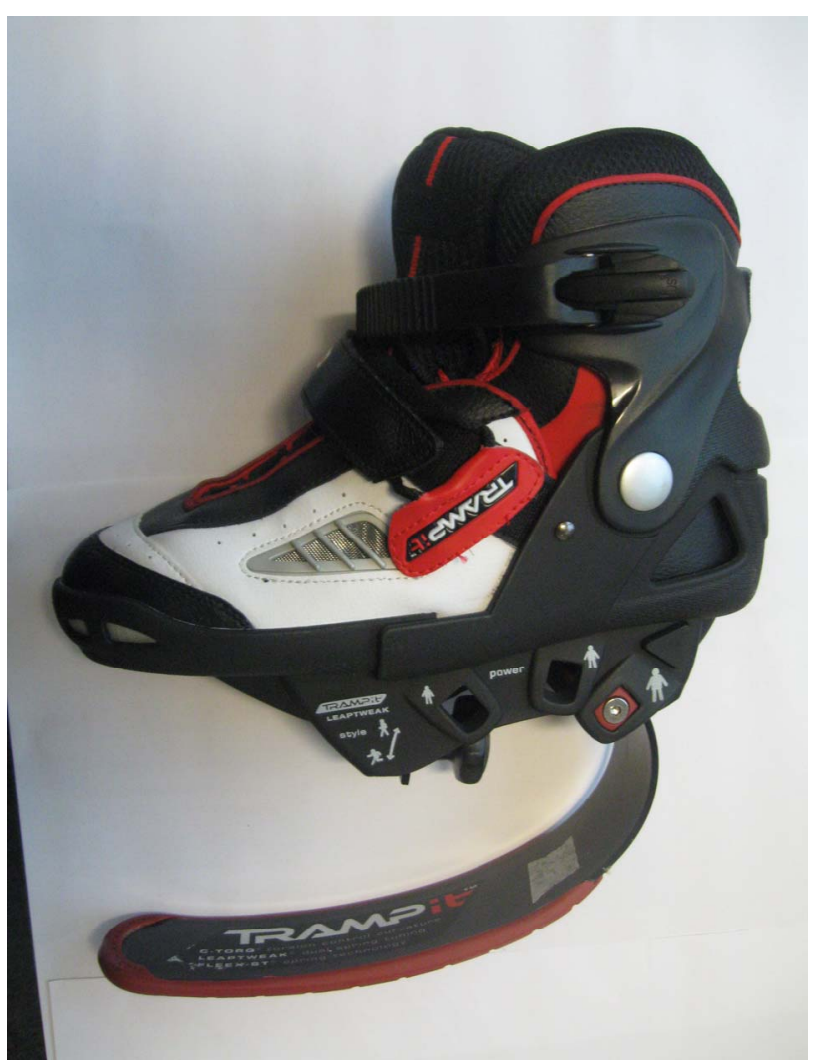

Figure 2. Energy return footwear. 
technology rather than be a direct equivalent replacement for sprint prosthesis.

\subsection{Linearity Characteristics of Energy Return Prosthesis}

A pilot study is conducted to investigate the linearity of energy return prosthesis. This will note its behaviour under load in lieu of the fact that no formalised test currently exists for sports prosthesis technology.

The prosthesis is loaded using a Testometric strength testing machine (Testometric Company Ltd., Lancashire, UK). The test prosthesis is shown in Figure $\mathbf{1}$ and the test machine set up is shown in Figure 3.

Two different methods of prosthesis loading are undertaken in this experiment. A schematic of the two conditions is shown in Figure 4.

1) A $28 \mathrm{~mm}$ slide of the distal end before becoming fixed at the distal end (SDE);

2) Fixed at the distal end (FDE).

Condition "a" demonstrates a compression of the prosthesis but the distal end is initially free to move under load. It does so for a fixed distance of $28 \mathrm{~mm}$ after which it then locks into position and continues to be loaded. Condition "b" demonstrates a prosthesis compressive load method whereby both the shank and the distal end are fixed.

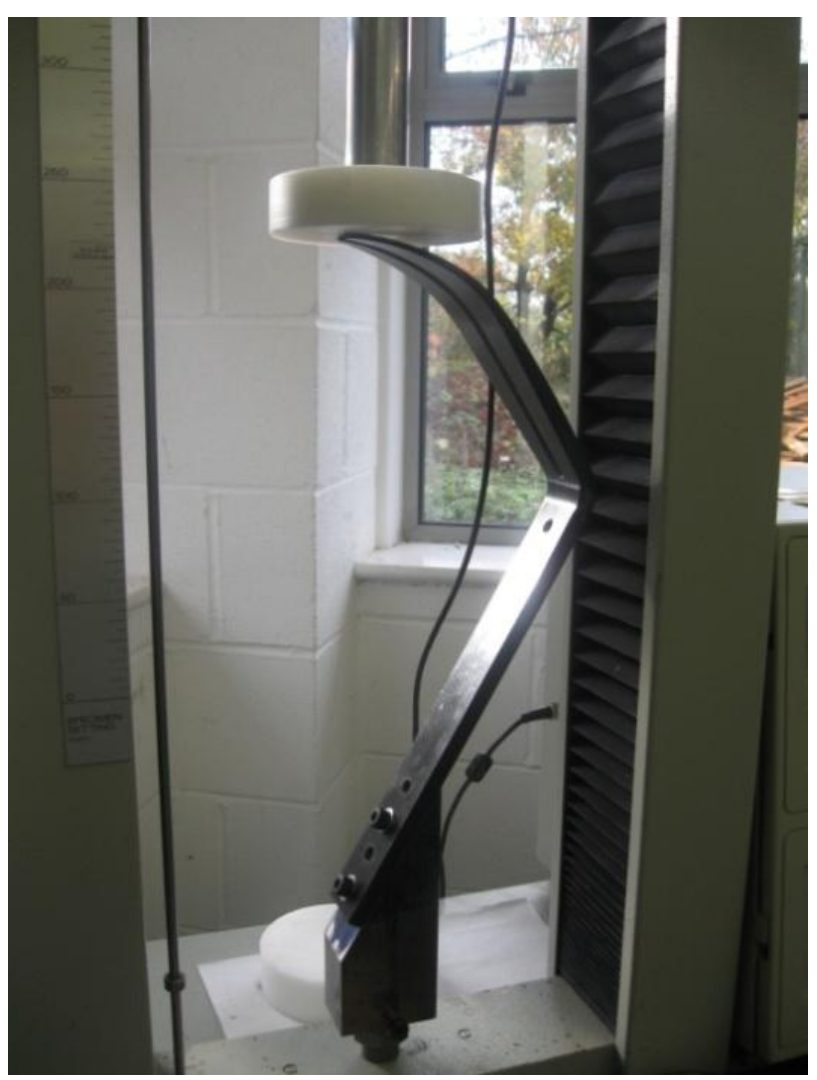

Figure 3. Prosthetic blade loading.

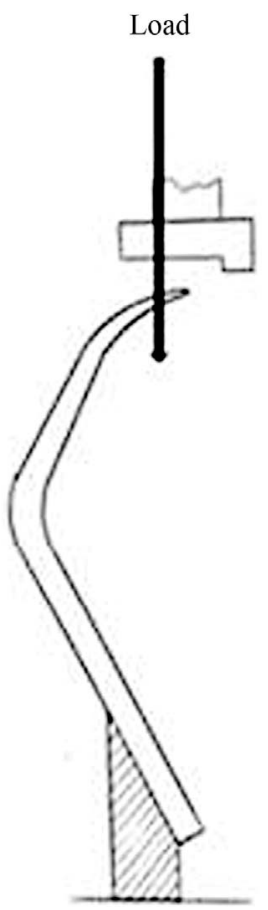

(a)

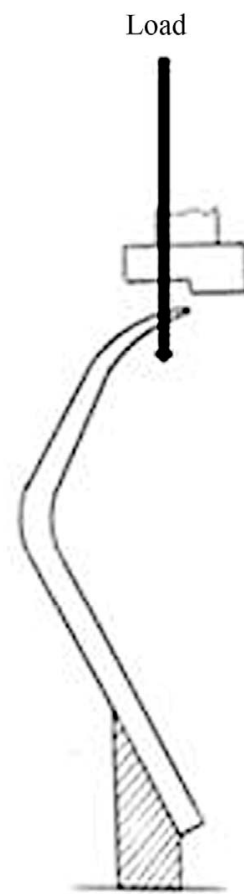

(b)
Figure 4. Testometric loading conditions for: (a) SDE; and (b) FDE method.

The prosthesis distal end of both conditions is fixed by locating against a ledge within an acetyl block as the load is applied. Ten loadings of each condition to a maximum load of $2000 \mathrm{~N}$ are conducted. $2000 \mathrm{~N}$ is used as this is approximately four times the bodyweight of the intended user for this prosthesis specification. Such a bodyweight impact has been suggested as being consummate of high speed running [11]. The mean of each loading is recorded and the coefficient of variation (CV) is used to ensure statistical repeatability and stability of the process. The CV is defined as Standard Deviation divided by the mean then multiplied by 100 to reflect this ratio as a percentage. The load application rate of each loading was $50 \mathrm{~mm}$ per minute.

Mechanical stiffness is calculated as load $(\mathrm{N})$ divided by deflection (mm). The stiffness's of both the peak loading and the average of the full load cycle was recorded.

\subsection{Prosthesis Drop Tests}

The energy return foot prosthesis used in test (a) is also affixed to an assembly comprising $18 \mathrm{Kg}$ of total mass. This mass/prosthesis assembly is dropped from a fixed height to observe the impact related response. The drop test release mechanism uses a high strength magnet with its polarity which can be deactivated by turning a dial. This minimises any unwanted motion of the prosthesis prior to release. The test rig can be seen in Figure 5. 


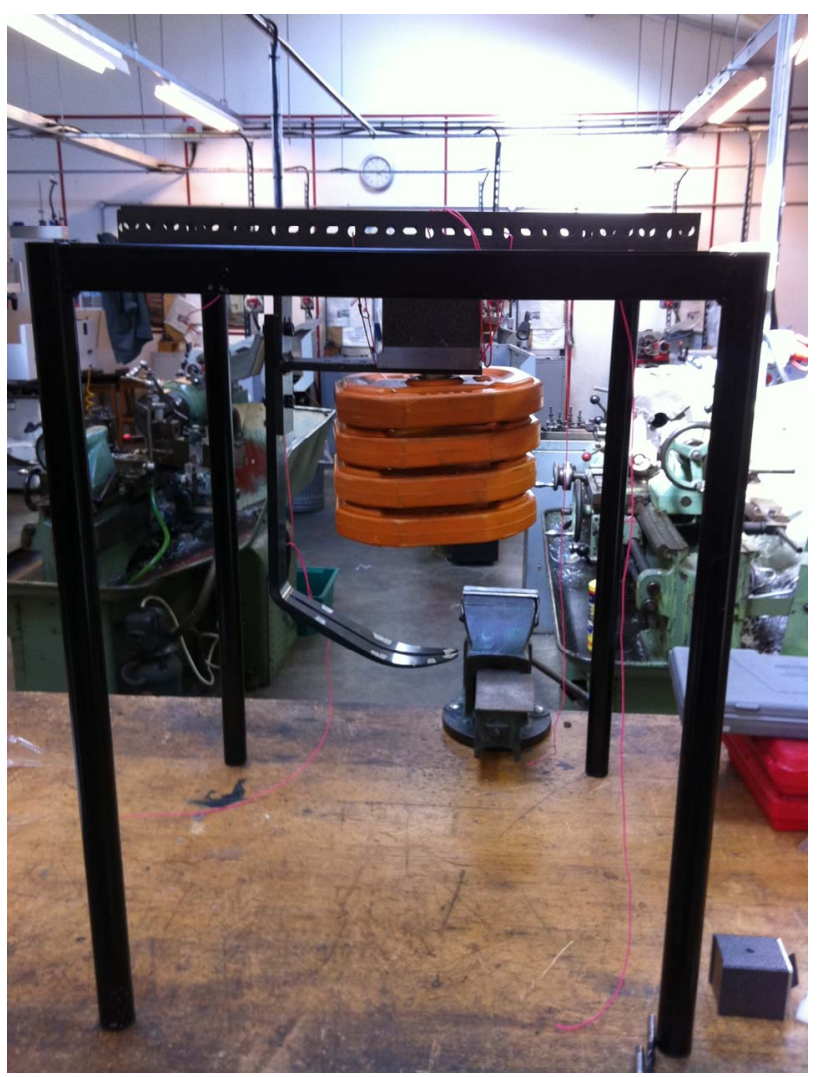

Figure 5. Prosthesis drop test rig.

Slippage of the prosthesis upon landing is minimised by using a high friction coefficient rubber affixed across the landing area. The falling trajectory of the prosthesis is tracked via 3 light reflective markers. Their motion is recorded using a digital video recorder filmed at a frequency of $210 \mathrm{~Hz}$. The light reflective markers are placed:

- On the top face of the prosthesis "toes".

- At the arbitrary "ankle" position of the prosthesis.

- At the straight, "shank" of the prosthesis.

The data is recorded from the period of prosthesis release to ground impact and then the rebound of the prosthesis. 10 "drops" were performed for this experiment from a height of $110 \mathrm{~mm}$. The area of interest for this experiment is the behaviour of the prosthesis and its light reflective markers as it contacts the ground.

The experiment is evaluated within the "Quintic" motion capture software (Quintic Consultancy Ltd. Coventry, UK) with further statistical evaluation performed using the exported raw data. The data is smoothed using software based Butterworth filters. Each light reflective marker is tracked for their movement in both vertical and horizontal directions during the drop test.

\subsection{Energy Return Technology Run Tests}

A series of steady state running test trials were performed to simulate the dynamic loading behaviour of energy re- turn technology. A non-disabled participant performed the trials. The participant was a current amateur athlete with a history of competitive running participation in events ranging from $100 \mathrm{~m}$ upto the marathon distance. They performed a self-selected warm up prior to the tests and gave written consent for this experiment.

Two different stiffness settings of the footwear were used. This was achieved by a fixed change in the blade length of each shoe. These were both run under a selfperceived speed by the participant which was requested to be as fast as they could feasibly achieve. In total, 7 trials of each condition were undertaken meaning for the purposes of this study, 14 runs in total were completed.

The run tests were conducted within an indoor, dry environment in the same session. The running area was segregated using tape into 3 distinct zones. There was an initial 15 metre zone used for the participant to accelerate from rest, a 4 metre zone whereby the participant was asked to ensure their best maximal but steady state speed, and finally another 15 metre zone used for the participant to safely rested-accelerate.

The running order of each trial was to perform 7 fast of the less stiff shoe setting and then 7 fast of the shoes stiffer setting. Whilst the stiffer blade setting of the shoe was achieved by shortening the blade, this actually changed the geometry of the blade and increased the shoes total height by $55 \mathrm{~mm}$ when unloaded. Each trial was designated:

1) Symmetrical set up, less stiff blade, "fast" effort;

2) Symmetrical set up, stiffest blade setting, "fast" effort.

The trials were filmed using a single video camera positioned opposite the steady state run zone and were filmed at a frequency of $210 \mathrm{~Hz}$.

The information of interest in this trial was the steady state speed achieved the stride length, limb to limb symmetry and a qualitative visual examination of the shoe blade behaviour. The visual examination of the trials was undertaken by reviewing the video footage.

\section{RESULTS}

\subsection{Linearity Characteristics of Energy Return Prosthesis}

The results of the FDE and SDE loading conditions are shown in Table 1.

The low coefficient of variation suggests extremely high levels of repeatability of the prosthesis behaviour using both the FDE and SDE methods. The SDE method had a CV of $0.1 \%$. The SDE method had a CV of $1.7 \%$. The obtained stiffness from the two methods does highlight a distinctive difference in measured performance. Because deflection measured by the assessment machine will be relative, it is obvious to see a difference in the 
Table 1. Loading method results.

\begin{tabular}{lcc}
\hline \multicolumn{1}{c}{ Value } & FDE & SDE \\
\hline Mean stiffness of load cycle (N/mm) & 48 & 42 \\
Average stiffness of total load cycle (N/mm) & 39 & 26 \\
Peak stiffness from 1500 N - 1950 N sample (N/mm) & 69 & 76 \\
$\begin{array}{lcc}\text { Percentage increase of peak load stiffness over average } \\
\text { stiffness (\%) }\end{array}$ & 23 & 61 \\
CV of stiffness at 1950 N (\%) & 1.1 & 0.2 \\
CV of average stiffness of total load cycle (\%) & 1.7 & 0.1 \\
\hline
\end{tabular}

overall mean. However, there is also a difference when measuring the last $450 \mathrm{~N}$ loading sample too.

The typical load/deflection plots of both the FDE and SDE methods can be seen in Figure 6.

It can be seen that allowing a slide of the distal end does create a significantly different obtained stiffness. The FDE method has a higher overall mean stiffness. The SDE method is less stiff as the changing spring length is altering the load cycles boundary conditions and thus its mechanical properties.

From these plots it can also be seen that the prosthesis exhibits initial non-linear behaviour irrespective of which loading methods are used. The SDE method does show a decrease in stiffness caused by the controlled distal end drift which will be due to the relative measurement of the machine. However, once engagement of the distal end takes place, a reduced, progressive non-linearity is witnessed and a near parallel trace of the two methods takes place. However, whilst it appears identical, the SDE and FDE mechanical stiffness of the upper $450 \mathrm{~N}$ final load cycle of the graph trace does still have a slight difference (as shown in Table 1).

\subsection{Prosthesis Drop Tests}

The $1018 \mathrm{Kg}$ drop tests produced repeatable behavior of the experiment achieving a coefficient of variation of the dimensional movement of the light reflective markers of $9 \%$. The typical behavior of the drop test by both the toe and shin markers is shown in Figure 7.

Figure 7 demonstrates that upon ground contact, the prosthesis began to rotate forwards (clockwise) approximately about the toes. The deviation from $0 \mathrm{~mm}$ of the markers during initial freefall was caused by a slight sheering of the prosthesis away from the release magnet when dropped. Upon impact, the toe marker exhibited a direct response to the impact by moving horizontally in a negative direction $2 \mathrm{~mm}$ in 0.04 seconds.

The area of interest to ascertain any change in ground contact boundary conditions created by the ground impact is the horizontal movement of the toe marker. A magnification of the toe marker horizontal displacement at the point of ground impact is shown in Figure 8.

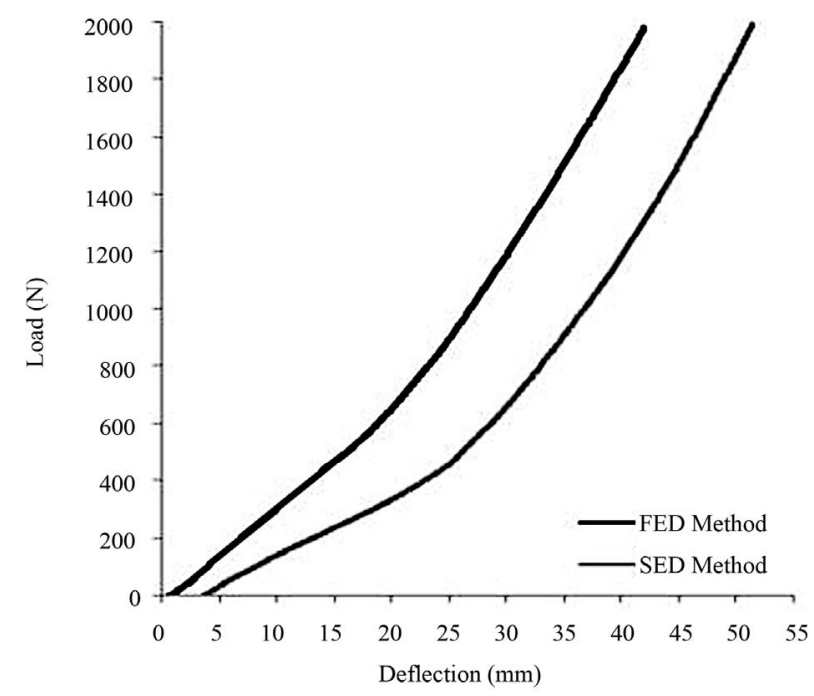

Figure 6. FDE \& SDE load/deflection behaviour.

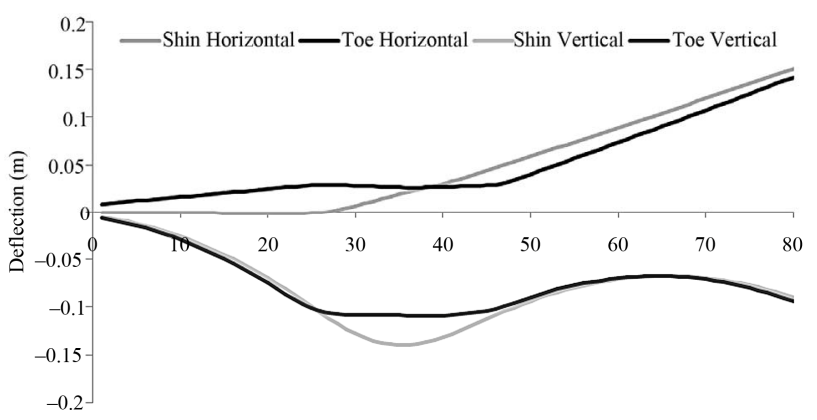

Figure 7. Drop test behavior.

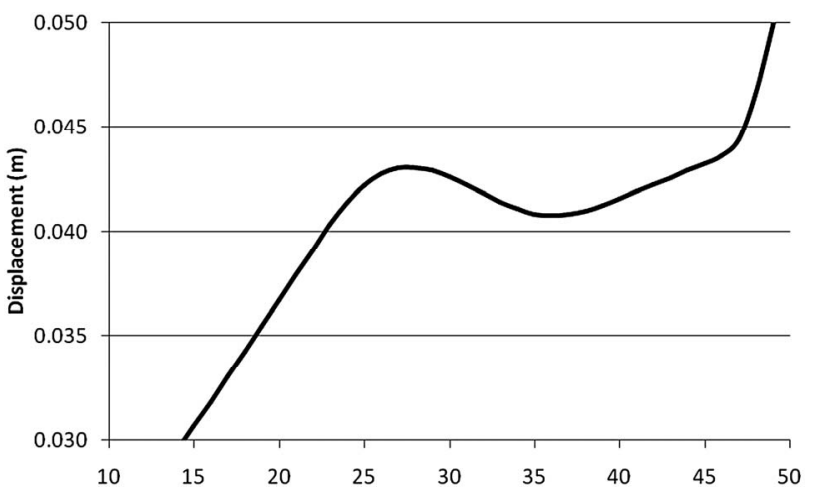

Figure 8. Toe marker horizontal impact displacement.

It can be seen in Figure 8 that immediately after ground impact occurs, there is a sudden negative horizontal change in displacement of $2 \mathrm{~mm}$. At the same point in time, the "ankle" marker did not move in the horizontal plane. This suggests that the energy return blade bent at the point of impact rather than due to any rotation of the prosthesis to achieve it.

The graph in Figure 8 shows that ground impact takes place at approximately time frame 25. Upon impact, a slight rotation of the prosthesis and mass occurs upon 
release. The vertical displacement of the shin maker shows no value until ground contact. At which point the blade compresses and the prosthesis begins to rotate forwards as well as the energy transferred by the impact is then returned to the blade resulting in an upward launch trajectory.

The toe marker also was subjected to a positive vertical displacement of $2 \mathrm{~mm} 0.01$ seconds after the point of impact. This demonstrates that the ground point of impact was likely slightly behind the location of the toe marker. The $2 \mathrm{~mm}$ measured vertical displacement reaction is the toes "curling" upwards due to the impact force. The prosthesis unloaded length compressed by approximately $30 \mathrm{~mm}$ upon ground impact.

\subsection{Energy Return Technology Run Tests}

The results showing the conduct of the trials are shown in Table 2.

The Coefficient of Variation scores are of a low percentage especially considering the subjective nature of the running speeds by the participant.

At the point of contact the blade begins to compress. As the participant approaches mid stance, it can be seen that the distal end of the blade moves vertically towards the sole as the blade compresses.

At the point of ground contact, the blade contacts the ground roughly midfoot of the blade which then transfers marginally to the rearfoot due to the blade bending. However, once mid stance is achieved, the point of contact begins to shift forwards until take-off. This will mean that the effective spring length of the blade at the beginning of the gait cycle is quite short but then progressively lengthens towards toe-off. This demonstrates a non-linear response in stiffness from a blade of this nature both under compression and extension. Ultimately the blades stiffness will reduce as the ground contact phase continues and potential energy is converted to kinetic energy through vertical movement of the athlete. Enlarged and overlaid images of the ground contact phases are shown in Figures 9(a) and (b).

Figure 9 demonstrates ground contact point shifting due to running gait. In both images, point 1 indicates the initial ground contact point. The arrow in Figure 9(a) shows the shift from heel strike to mid stance. The arrow demonstrates the mid stance distal end of the blade upward deflection. Figure 9(b) shows the ground point of contact shift from heel strike (1) to toe off (2). The arrow demonstrates the displacement of the boots ankle point from initial contact to toe off.

\section{DISCUSSION}

The first research question asked if static load assessment was suitable to measure lower-limb foot prosthesis used for competitive running. The two different loading techniques used in this pilot study did not support this. Primarily there is a difference in performance of the prosthesis depending on its length or contact point. Nonlinearity was witnessed in the early stages of loading. It is proposed that this is due to the tapered profile of the "foot" region of composite material. The magnitude and proportion of such non-linearity would likely be small and unique to each design but it should be reflected that such a characteristic exists. This supports previous claims that variable stiffness parameters could be important for running prosthesis in the future [12].

The second research question asked whether a prediction of energy return technology was possible using static load techniques. With the SDE method, the obtained bending deflection would be inaccurate due to the constantly shortening spring length of the "toes" arching through. The $28 \mathrm{~mm}$ slippage of the SDE method created a $12 \%$ perceived loss in prosthetic stiffness. This was caused by a combination of the change in spring length and the relative measurement of compressed deflection of the machine. Such a characteristic does make the prediction of ERP stiffness by extending the linear portion more inaccurate and therefore unfair to assess or regulate the technologies response. From a clinical point of view, not ensuring the ground contact point and the point statically loaded are the same could mean that at best, significant tuning of an athlete's prosthesis geometry would be required and at worst that an incorrect prosthesis would be fitted. If such a technique was used to prescribe or evaluate ERP technology in the future, the lower portions of such graph traces should be disregarded and the linear-like section of a load as close to those expected in the individual's event should be selected.

The final research question in this pilot study asked whether LLP's are subjected to significant boundary condition changes due to loading. The pilot tests here would suggest that they are. The drop test produced a change in boundary conditions due to impact load and a resultant deflection which would alter the blades stiffness. A boundary change was produced due to a change in the

Table 2. Condition 1 \& 2 summary.

\begin{tabular}{cccccc}
\hline & Blade Setting & Self-Selected Speed & $\begin{array}{c}\text { Running Speed } \\
\text { Mean (M/s) }\end{array}$ & $\begin{array}{c}\text { Mean Stride } \\
\text { Freq. (Hz) }\end{array}$ & $\begin{array}{c}\text { Stride Freq. Coefficient of } \\
\text { Variation (\%) }\end{array}$ \\
\hline $\mathbf{1}$ & Less stiff & "Fast” & 4.60 & 2.98 & 7 \\
$\mathbf{2}$ & More stiff & "Fast” & 4.68 & 3.33 & 5 \\
\hline
\end{tabular}




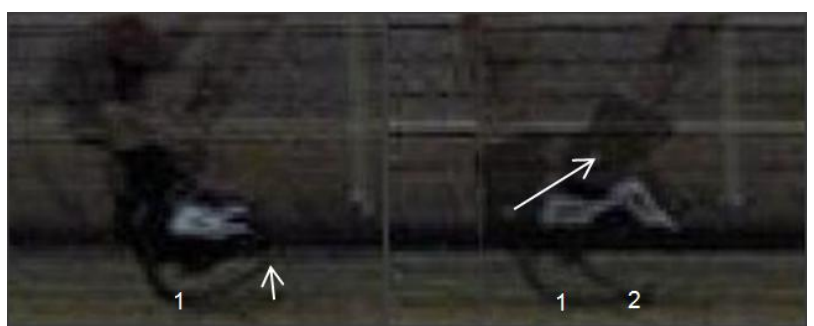

Figure 9. (a) Heel strike to mid stance; (b) Heel strike to toe off.

ground contact point due to impact. Upon impact, the prosthesis bent at a position somewhere between the "ankle" and "toe" markers causing only the toe marker to produce a negative horizontal displacement. The shin marker was not subjected to any horizontal displacement at the exact point of impact. However, because the ERP was designed for a user of $\sim 55 \mathrm{Kg}$, the limitations of only using an $18 \mathrm{Kg}$ drop mass produced a deflection far less pronounced than would be desirable. The lighter mass was used due to the limits of the magnetic force used to hold the prosthesis prior to drop.

The run tests produced a change in boundary conditions due to deflection and stiffness variation of the blade due to foot roll. However, unlike the drop tests, this was due to the amount of clockwise rotation the blade was subjected to during the gait cycle. Further investigation is required to ascertain the magnitude of foot roll in amputee elite athletes.

The runs produced a step frequency of around $3 \mathrm{~Hz}$ which is less than the reported $5 \mathrm{~Hz}$ witnessed in ablebodied $100 \mathrm{~m}$ sprinting [11]. However, such a comparable effort would have meant excessive fatigue on the part of the participant, safety concerns using the shoe technology at such speed and the larger acceleration and deacceleration zones required.

The results of these pilot trials do have limitations but do suggest that further assessment of variable dynamic stiffness of lower-limb ERP's using amputee candidates are now warranted.

\section{CONCLUSION}

Several pilot studies were undertaken to investigate the behavior of energy return lower-limb technology used for elite level high speed running. Through these initial investigations, it was concluded that static load testing is not recommended to predict, specify, or regulate such technology. The outcome of doing so would produce inaccurate performance and unfair thresholds in performance being calculated. It was demonstrated that energy return prosthesis are subject to changes in mechanical stiffness due to ground contact deflection or gait induced changes in effective prosthetic blade length. This pilot study suggests that a linear response of prosthetics energy return technology when running should not be assumed. As a result, further investigation into the dynamic behavior of lower-limb prosthesis is warranted.

\section{ACKNOWLEDGEMENTS}

The prosthesis used in this study was kindly donated by Chas A Blatchford \& Sons Ltd. (Basingstoke, UK). Special thanks go to both Shelley Broomfield and Andrew Callaway for their assistance with the data collection of the run test experiments.

\section{REFERENCES}

[1] Hafner, B., Sanders, J., Czerniecki, J. and Fergason, J. (2002) Trans-tibial energy-storage-and-return prosthetic devices: A review of energy concepts and a proposed nomenclature. Journal of Rehabilitation Research and Development, 39, 1-11.

http://www.rehab.research.va.gov/jour/02/39/1/Hafner.ht $\underline{\mathrm{m}}$

[2] Nolan, L. (2008) Carbon fibre prostheses and running in amputees: A review. Foot and Ankle Surgery, 14, 125129. doi:10.1016/j.fas.2008.05.007

[3] Dyer, B., Redwood, S., Noroozi, S. and Sewell, P. (2011) The fair use of lower-limb running prostheses. Adapted Physical Activity Quarterly, 28, 16-26.

[4] Dyer, B., Noroozi, S., Sewell, P. and Redwood, S. (2010) The design of lower-limb sports prostheses: Fair inclusion in disability sport. Disability and Society, 25, 593602. doi:10.1080/09687599.2010.489309

[5] Weyand, P., Sternlight, D., Bellizzi, M. and Wright, S. (2000) Faster top running speeds are achieved with greater ground forces not more rapid leg movements. Journal of Applied Physiology, 89, 1991-1999.

[6] Arampatzis, A., Bruggemann, G. and Metzler, V. (1999) The effect of speed on leg stiffness and joint kinematics in human running. Journal of Biomechanics, 32, 13491353. doi:10.1016/S0021-9290(99)00133-5

[7] Brughelli, M. and Cronin, J. (2008) A review of research on the mechanical stiffness in running and jumping: Methodology and implications. Scandinavian Journal of Medicine \& Science in Sports, 18, 417-426. doi:10.1111/j.1600-0838.2008.00769.x

[8] McMahon, T. and Cheng, G. (1990) The mechanics of running: How does stiffness couple with speed? Journal of Biomechanics, 23, 65-78. doi:10.1016/0021-9290(90)90042-2

[9] Bruggemann, P., Arampatzis, A., Emrich, F. and Potthast, W. (2008) Biomechanics of double transtibial sprinting using dedicated sprinting prostheses. Sports Technology, 1, 220-227. doi:10.1002/jst.63

[10] McGowan, C., Grabowski, A., McDermott, W., Herr, H. and Kram, R. (2012) Leg stiffness of sprinters using running-specific prostheses. Journal of the Royal Society Interface, 9, 1975-1982. doi:10.1098/rsif.2011.0877

[11] Mero, A., Komi, P. and Gregor, R. (1992) Biomechanics 
of sprint running. Sports Medicine, 13, 376-392. doi:10.2165/00007256-199213060-00002

[12] Farley, C. and Gonzalez, O. (1996) Leg stiffness and stride frequency in human running. Journal of Biomechanics, 29, 181-186. doi:10.1016/0021-9290(95)00029-1 